\title{
Same Total Normal Forms Sperm Counts of Males From Lhasa and Shanghai, China
}

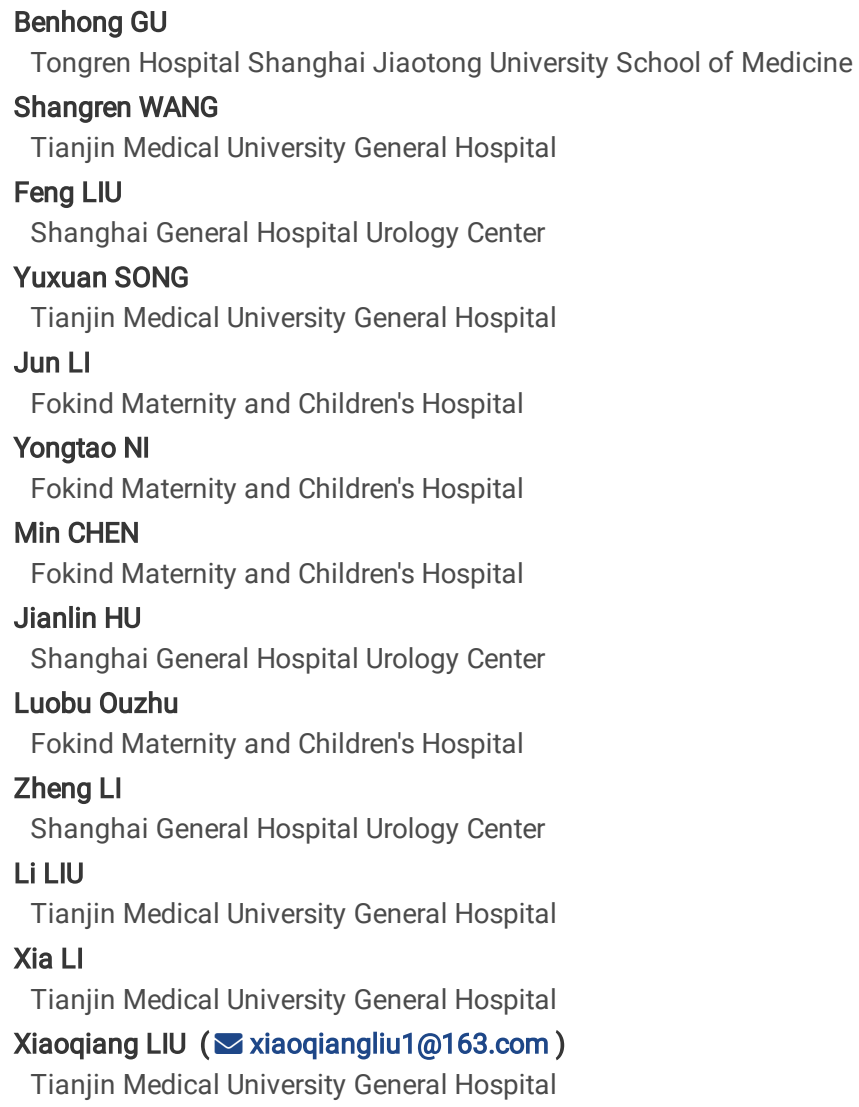

\section{Research Article}

Keywords: Plateau hypoxia, male infertility, normal forms, sperm count, sperm concentration, asthenozoospermia

Posted Date: June 7th, 2021

DOI: https://doi.org/10.21203/rs.3.rs-511098/v1

License: (;) This work is licensed under a Creative Commons Attribution 4.0 International License. Read Full License

Version of Record: A version of this preprint was published at Environmental Science and Pollution Research on October 26th, 2021. See the published version at https://doi.org/10.1007/s11356-021-17083-4. 


\section{Abstract}

Male infertility may be caused by genetic and/or environmental factors that impair spermatogenesis and sperm maturation. High-altitude (HA) hypoxic environments represent one of the most serious challenges faced by humans that reside in these areas. To assess the influence of the plateau environment on semen parameters, 2,798 males, including 1,111 native Tibetans and 1,687 Han Chinese individuals living in the plains(HCILP) who underwent prepregnancy checkups, were enrolled in this study. The semen samples of males were evaluated to determine conventional sperm parameters, sperm morphology, and sperm movement. Reproductive endocrine hormones (REHs) were detected in 474 males, including 221 Tibetans and 253 HCILP. Due to recurrent abortions in partners, the DNA fragmentation index (DFI) of 133 native Tibetans and $393 \mathrm{HCILP}$ individuals was further compared. Luteinizing hormone (LH) $(4.94 \pm 2.12$ vs. $3.29 \pm 1.43 \mathrm{U} / \mathrm{L})$, prolactin $(11.34 \pm 3.87$ vs. $8.97 \pm 3.48 \mathrm{nmol} / \mathrm{L}), \mathrm{E} 2 / \mathrm{T}(0.22 \pm 0.11$ vs $0.11 \pm 0.05)$, median total sperm motility (61.20\% vs. $51.56 \%)$, and DFI (23.11\% vs. $7.22 \%$ ) were higher in males from plateau areas while median progressive motility (PR) (35.60\% vs. $41.12 \%)$, total number of PR sperms (51.61 vs. $59.63 \mathrm{mil} /$ ejaculate), percentage of normal form sperms (3.70\% vs. $6.00 \%)$, curvilinear velocity (36.10 vs. $48.97 \mu \mathrm{m} / \mathrm{s})$, straight-line (rectilinear) velocity ( 14.70 vs. $31.52 \mu \mathrm{m} / \mathrm{s})$, estradiol (103.82 $\pm 45.92 \mathrm{vs} .146 .01 \pm 39.73 \mathrm{pmol} / \mathrm{L})$, progesterone (0.29 $\pm 0.27 \mathrm{vs}$. $2.22 \pm 0.84$ $\mathrm{nmol} / \mathrm{L}$ ), testosterone $(4.90 \pm 1.96 \mathrm{vs} .14 .36 \pm 5.24 \mathrm{nmol} / \mathrm{L})$, and testosterone secretion index (ratio of testosterone to $\mathrm{LH})(33.45 \pm 22.86 \mathrm{vs} 145.78 \pm 73.41)$ were lower than those in males from the plains. There was no difference in median total sperm number $(157.76 \mathrm{vs}$. $151.65 \mathrm{mil} / \mathrm{mL}), \mathrm{sperm} \mathrm{concentration}$ (52.40 vs. $51.79 \mathrm{mil} / \mathrm{mL}$ ), volume (3.10 vs. $3.10 \mathrm{~mL}$ ), total normal form sperms (5.91 vs. 6.58 mil/ejaculate, p50), and follicle-stimulating hormone (FSH) levels ( $4.13 \pm 2.55 \mathrm{U} / \mathrm{L}$ vs $3.82 \pm 2.35 \mathrm{U} / \mathrm{L})$ between the two groups of males. The REH and sperm parameters of males from HA hypoxic environments were adaptively altered. Although the total number of PR sperm decreased and DFI increased, the Tibetan population that lives at HAs has been found to grown continuously and rapidly. These results supplement prior findings regarding the impact of HA on male reproductive function.

\section{Introduction}

Infertility is a global health problem that is affecting more than 70 million couples worldwide (Nikshad et al., 2021). Although male infertility directly accounts for approximately $30 \%$ of couple infertility, it is estimated to be a cofactor in an additional $40 \%$ of all infertility problems (Del Vento et al., 2018). The Tibetan Plateau, with an average elevation of more than $4,000 \mathrm{~m}$, represents one of the most extreme environments for human settlement due to severe hypoxia at high altitudes (HAs). Humans have had to develop physiological adaptations to cope with the extreme environments at HAs. Presently, approximately 5 million indigenous Tibetans live on the plateau and have adapted well to the HA hypoxic environment (Qi et al., 2013). In humans (Weitz, 1981) and domestic animals, such as sheep, that live in HA environments caused by high plateaus (Parraguez et al., 2006), the fertility of females was found to be reduced relative to that of their low-altitude counterparts. Multiple meta-regression analysis of males residing on plains reported a significant decline in sperm counts between 1973 and 2011 (Levine et al., 2017). However, the effect of HA on semen parameters and its underlying mechanism are currently unclear.

The impact of HA hypoxic environment on sperm quality has been suggested by several studies. However, the results are still controversial. An HA environment has been demonstrated to cause a decline in semen quality. Short-term HA exposure for more than one month at an elevation of $3,600 \mathrm{~m}$ increases the distribution of sperm deformities (Zheng, Liu, Li, \& Tian, 2019). Exposure to hypoxia at HA (5,380 m) for 1 year induces reversible effects on semen quality and serum reproductive hormones levels in young male adults. At HAs, the sperm motility rate decreases (He et al., 2015). Meanwhile, the levels of testosterone ( $\mathrm{T}$ ) and estradiol (E2) also decline (Okumura, Fuse, Kawauchi, Mizuno, \& Akashi, 2003). After staying in HA regions for approximately half a year, the levels of follicle-stimulating hormone significantly increase while those of luteinizing hormone (LH), prolactin (PRL), and T significantly decrease.

In previous studies, no significant difference was observed between the sperm concentrations, straight-line velocity (VSL), percentage of morphologically normal sperms, total T concentration, or FSH levels between normal fertile males (33 native Tibetans vs. 23 immigrated Han Chinese who stayed at HAs for over 3 years). However, the immigrated Han Chinese had higher sperm motility, lower curvilinear velocity (VCL), and lower LH levels than native Tibetans. Moreover, no difference was found in the sperm DNA fragmentation index (DFI) between native Tibetans and immigrated Han Chinese (Gu et al., 2018). To date, no study has evaluated the impact, if any, of HA on sperm parameters in males who have been residing in the Qinghai-Tibet plateau for years. Therefore, this study was carried out to evaluate the differences in conventional sperm parameters, reproductive endocrine hormones (REHs), and DFI between males who have been living in plateau and plain regions for many years.

\section{Methods}

\section{Patient selection}

This retrospective study was performed using the databases of the Department of Andrology, Center of Urologic Medicine, Shanghai General Hospital affiliated to Shanghai Jiao Tong University (Shanghai, China), and Tibet Fukang Women and Children's Hospital (Lhasa, China). Data for males who underwent pre-pregnancy checkups from May 2015 to July 2019 were collected. The inclusion criteria were: (1) males who were born and have been residing in Lhasa (group of plateaus) and Shanghai (group of plains) for many years and (2) males who underwent sperm analysis in labs. Patients with oligozoospermia (total sperm count $<39 \mathrm{mil} / \mathrm{mL}$, but $>0$ ), asthenozoospermia (percentage of progressive motility $(\mathrm{PR})<32 \%$ ), or teratozoospermia (percentage of normal forms (NFs) < 4\%) were advised to undergo REH and DFI detection analyses. Finally, a total of 2,798 males who were unaware of their fertility status and provided a nonazoospermic semen sample were included in the analysis. Finally, 474 males, 221 native Tibetans and 253 HCILP, underwent REH detection. DFI was detected in 133 native Tibetans and 393 HCILP. The exclusion criteria of this study were: history of genetic abnormalities, systemic diseases, hormonal treatment, alcohol or drug abuse, male accessory gland infection, varicocele, and cryptorchidism. Patients whose medical anamnesis was unclear or incomplete were excluded from the study.

\section{Sperm analysis}


Semen samples from males were obtained via masturbation in a room close to the andrology laboratory and the laboratory of the reproductive medicine center. Males were asked to abstain from ejaculation for 2-7 days before sampling. Abstinence time was calculated based on the self-reported time of the previous ejaculation and the time of sample collection. Semen samples were analyzed in accordance with the current World Health Organization (WHO) guidelines (Cooper et al., 2010), as previously described (Jorgensen et al., 2012). The reports indicated whether the sample collection was complete. If incomplete, samples were collected again after 2-7 days of abstinence. Semen samples from Lhasa and Shanghai males were analyzed for semen volume, sperm concentration, total sperm count, and sperm motility. The Kruger strict morphological standard, mentioned below, was used for sperm morphology analysis: 1) the sperm head should be oval in shape with a smooth surface and regular outline, 3.7-4.7 $\mu \mathrm{m}$ in length, and 2.5-3.2 $\mu \mathrm{m}$ in width; 2) the acrosome accounts for approximately $40 \%-70 \%$ of the head area; the structure should be clear with no large vacuoles in the acrosome area, no more than two small vacuoles should be present, and the area of vacuoles should not be more than $20 \%$ of the sperm head area; 3 ) the middle part of sperm tail should be slender and regular, similar to the length of sperm head; the longitudinal axis of the middle part should be aligned with the longitudinal axis of sperm head; the cytoplasmic remnant is considered abnormal only when it is larger than one third of the sperm head; 4) the length of the main tail segment should be approximately $45 \mu \mathrm{m}$, which is longer than the length of the middle segment and 10 times longer than that of the head. If there is no sharp angle of flagellum rupture, the end of the flagellum can freely curl into a ring. Oligozoospermia was defined as a total sperm count of $<39$ million/ejaculate, asthenozoospermia was defined as percentage of PR spermatozoa $<32 \%$, and teratozoospermia was defined as percentage of morphologically normal spermatozoa $<4 \%$.

\section{Reproductive hormones}

Venous blood samples were withdrawn from the participants in the morning on the day of participation, and serum was isolated and stored at $-20{ }^{\circ} \mathrm{C}$. The Roche E601 automatic chemiluminescence analyzer (Roche China, Shanghai, China) was used to detect the levels of sex hormones, including T, FSH, LH, progesterone, PRL, and E2. All reagents, standards, and quality control materials were provided by Biovan company (Shanghai, China); all materials were used in strict accordance with the manufacturer's instructions.

\section{DFI}

Evanson's method is considered to be the gold standard for DFI detection and has been widely used worldwide (Alharbi, Hamouche, Phillips, Kadoch, \& Zini, 2019). In our study, the human sperm nuclear integrity staining kit (Xingbo Biological, Shanghai, China) was used to detect DFI (Du et al., 2017). The chromatin structure of damaged sperm is loose; thus, the DNA denatures to form a single strand under the action of acid while the DNA of normal sperm maintains the double-stranded structure. Acridine orange (AO) emits green fluorescence on combination with double-stranded DNA and yellow or red fluorescence on combination with single stranded DNA. Sperms were stained with AO and analyzed using flow cytometry. A certain amount of semen was diluted to $100 \mu \mathrm{L}$ with solution A to derive a final concentration of (1-2) $\times 10^{6}$ sperm cells $/ \mathrm{mL}$. After adding $200 \mu \mathrm{L}$ of solution B (cells were placed on ice for operation), $600 \mu \mathrm{L}$ of solution $\mathrm{C}$ was added after a certain time interval for $30 \mathrm{~s}$. At least 5,000 sperm cells from each sample were analyzed using BD Accuri ${ }^{\circ}$ C6 flow cytometer (BD Bioscience, Shanghai, China), and the results were analyzed using Accuri® CFlow ${ }^{\circledR}$ software (BD Bioscience, Shanghai, China).

\section{Quality control}

All experimental technicians participated in the standardized training of the WHO Laboratory Manual for human semen examination and processing (5th Edition), and the internal and external quality parameters of the laboratory met the specified requirements. Additionally, sperm morphology was examined using a sperm-smear, which was prepared by air drying, fixation, and Diff-Quik staining. Sperms were diluted and stored as standard samples of different concentrations for quality control. Sperm motility was reviewed using a videotape recorded on DVD. In each quality control experiment, each quality control project was analyzed with two different samples. In this study, all semen analyses were performed by two groups of experimental technicians (plateau group and plain group), who were trained and guided by the same teacher, and morphological smear test and DFI were evaluated by the same technician.

\section{Statistical analysis}

Data were analyzed using Stata version 14.1 (StataCorp LP, College Station, TX, USA). First, the semen parameters, REH and DFI, were tested for normality and homogeneity of variance, and subsequently, the test method was determined. Student's $t$ test was performed for data conforming to normal and homogeneity of the variance tests, otherwise Wilcoxon rank sum test was performed. If the count data were positively skewed, geometric mean and P5, P50, and P75 were used. The $\chi^{2}$ test was performed for the classified data. A $p$ value less than 0.05 was considered statistically significant.

\section{Ethics approval and consent to participate}

This study was approved by the Ethics Committee of Shanghai General Hospital affiliated to Shanghai Jiao Tong University and Tibet Fukang Women and Children's Hospital (31660308) and complied with all specified guidelines. The study was also conducted in accordance with the Declaration of Helsinki guidelines.

\section{Results}

\section{Comparison of semen quality}

To reveal the differences in semen quality between groups, 2,798 males (median age= 30 years old), including 1,111 native Tibetans and 1,687 HCILP, were included in the analysis (Table 1). According to the WHO standards, the incidence rate of normozoospermia, oligozoospermia, asthenozoospermia, and teratozoospermia was compared between the two groups. Overall, the incidence rate of teratozoospermia $(54.27 \% \mathrm{vs} 36.57 \%, p=0.000)$ and asthenozoospermia $(42.03 \%$ vs $32.84 \%, p=0.000)$ was higher in males from HA areas than in those from plain areas. However, normozoospermia was higher in males from plain areas than in those from plateau areas $(44.16 \%$ vs $26.46 \%, p=0.000)$. By stratifying the data by age (the young group: $16 \leq$ age $\leq 30$; the 
old group, $30<$ age $\leq 40)$, we found that the incidence rate of teratozoospermia $(50.68 \%$ vs $58.25 \%, p=0.007)$ in native Tibetans increased with age while the incidence of asthenozoospermia in HCILP decreased significantly $(44.31 \%$ vs $33.71 \%, p=0.000)$. In both groups, normozoospermia remained stable with age (Fig. 1).

\section{Comparison of semen parameters}

To comprehensively study the effects of an HA hypoxic environment on semen quality, we assessed and evaluated semen volume, sperm concentration, sperm motility, sperm morphology, total sperm number, total NFs, and total PR. No significant difference was observed in the semen volume, sperm concentration, total sperm number, and total sperm numbers of NFs between the groups; however, native Tibetans had a lower median $\mathrm{NF} \%$ ( $3.70 \% \mathrm{vs} 6.00 \%, p$ $=0.000)$, higher median total percentage of PR+NP $(61.20 \%$ vs $51.56 \%, p=0.000)$, lower median PR\% $(35.60 \%$ vs $41.42 \%, p=0.000)$, and lower total sperm number of PR (51.61 million vs 59.63 million, $p=0.008)$. At the same time, males from the plains showed higher VCL and VSL than those from plateaus.

The data were stratified by age, as mentioned before, to identify any changes in semen or sperm parameters with aging. We observed significant differences in $\mathrm{NF} \%$, total percentage of PR+NP, and PR\% between the young and old groups from different areas, as mentioned above. However, no difference was observed between the young and old groups from the same area. The total sperm number with PR was higher in young males from plains than in those from plateaus while no difference was observed in males who were in their 30s. After stratification, males from the plains displayed higher VCL and VSL than those from plateaus. Further, a significant decrease was observed with aging (Table 1).

\section{Comparison of REHs}

Spermatogenesis is carefully regulated by endocrine hormones. In total, 221 native Tibetans and 253 HCILP without hypergonadotropic hypogonadism or hypogonadotropic hypogonadism underwent REH detection. Although the native Tibetans were slightly older than HCILP (31.34 \pm 4.20 years vs $29.66 \pm 4.71, p$ $=0.000)$, their levels of E2 (103.82 \pm 45.92 vs. $146.01 \pm 39.73 \mathrm{pmol} / \mathrm{L}, p=0.000), \mathrm{T}(4.90 \pm 1.96 \mathrm{vs} .14 .36 \pm 5.24 \mathrm{nmol} / \mathrm{L}, p=0.000)$, and progesterone $(0.29 \pm$ 0.27 vs. $2.22 \pm 0.84 \mathrm{nmol} / \mathrm{L}, p=0.000)$ were lower than those of their counterparts. However, the levels of LH (4.94 $\pm 2.12 \mathrm{vs}$. $3.29 \pm 1.43 \mathrm{U} / \mathrm{L}, p=0.000)$ and $\mathrm{PRL}(11.34 \pm 3.87$ vs. $8.97 \pm 3.48 \mathrm{nmol} / \mathrm{L}, p=0.000)$ were higher. No difference was observed in the levels of FSH $(4.13 \pm 2.55 \mathrm{U} / \mathrm{L}$ vs $3.82 \pm 2.35 \mathrm{U} / \mathrm{L}, p=$ $0.095)$ between the two groups.

After stratification of the REH data, we found that the levels of E2, T, and progesterone were lower in native Tibetans than in HCILP; however, opposite findings were obtained for the levels of PRL and LH. The data showed that the levels of T and progesterone decreased in HCILP and those of PRL decreased in native Tibetans with aging. However, FSH levels increased in both groups while LH and E2 levels did not show a significant change with aging in the two groups (Table 2).

Men from the plateaus had a lower testosterone secretion index (TSI, ratio of T to LH) $(33.45 \pm 22.86$ vs $145.78 \pm 73.41, p=0.000)$ and higher E2 to T ratio than those from plains $(0.22 \pm 0.11$ vs $0.11 \pm 0.05, p=0.000)$. During aging, no significant change was observed in E2/T and TSI of native Tibetans. However, HCILP displayed a significant decrease in TSI.

\section{Comparison of DFI}

Upon examination of conventional semen parameters, the quality of semen, sperm concentration, and sperm vitality could be determined; however, the evaluation of sperm functions was limited. Thus, the sperm fertilization ability and impact on embryo development could not be directly determined. DFI is one of the most common indicators to evaluate sperm fertility and predict pregnancy outcome. We found that the DFI of native Tibetans was significantly higher than that of HCILP $(23.11 \%$ vs. $7.22 \%, p=0.000)$, and no significant change was observed in DFI with an increase in age in males younger than 40 years of age (Table 3).

\section{Discussion}

\section{Principle findings}

In this study, we showed that an HA hypoxic environment may affect the hypothalamus-pituitary-testis axis of males, resulting in a decrease in the levels of T, E2, and TSI and an increase in the levels of LH and E2/T. However, no significant effect was observed on the total number of normal form sperms.

Tibetans have established a relatively ideal adaptation mechanism to the plateau environment. Tibetans have resided in the Qinghai-Tibet plateau for thousands of years. The average altitude of the Qinghai-Tibet plateau is above 4,000 meters; thus, it is called "the roof of the world". HA regions have an extremely cold environment, high radiation, and hypoxia, which make them unlivable. Although nearly $70 \%$ of Tibetans live in areas above 3,500 meters (Wu, 2001), to better adapt to the plateau environment, Tibetans have established a relatively ideal genetic adaptation mechanism to be free from diseases caused by maladjustment (Childebayeva et al., 2019). Tibetans have been reported to experience a rapid expansion during the Neolithic period, suggesting that Tibetans adapted to the HA environment during this period (Qi et al., 2013). Despite the permanent stress induced by hypoxic exposure, humans in HA areas have a reduced cancer mortality over a broad spectrum of cancer types (Thiersch \& Swenson, 2018). Besides the social factors, there may be some physiological mechanism that helps them adapt to the HA environment. To date, the changes that may have occurred in terms of the reproductive capacity of males residing in the plateau remain unknown. In the present study, we collected data from life-long residents of Shanghai plains (altitude of 4 m) and Lhasa plateau (altitude of $3,650 \mathrm{~m}$ ) who underwent pre-pregnancy checkups. Thereafter, we retrospectively analyzed the conventional sperm parameters, REHs, and DFI.

Hypoxic exposure can result in a significant decrease in the reproductive capacity of animals and humans 
Following exposure to a simulated altitude, male rats present a significant decrease in testicular mass and the height and diameter of seminiferous tubules, increase in interstitial space, depletion of cellular elements, vacuolization in epithelial cells, and folding of the basal membrane (Cikutovic, Fuentes, \& BustosObregón, 2009; Farias et al., 2005). The positive rate of acrosin and in vitro incidence of acrosome reaction are reported to be significantly lower in rat sperms under hypoxic environments than in wild-type sperms, indicating that hypoxia can inhibit sperm capacitation and acrosome reaction, which may be related to sperm oxidative stress (Liao et al., 2010). Some researchers also concluded that hypoxia and transient ischemia can lead to local damage in seminiferous tubules (Guan, Zheng, Yang, \& Li, 2008) and degeneration of germinal epithelium (Salmasi et al., 2005). Such findings revealed that hypoxic exposure can result in a significant decrease in the reproductive capacity of animals.

Approximately 45 years ago, a German scientist, Eckes $\mathrm{L}$, found that the mean birth weight of males and animals decreased in HA regions while the mean placental weight was greater (relatively and absolutely) in HA regions due to the enlargement of capillary volume. The lower birth weight corresponds to a higher neonatal mortality, which increases with an increasing altitude (Eckes, 1976). To date, only few studies have assessed the semen parameters of longterm immigrants (mostly soldiers) at HAs (He et al., 2015). In healthy males, chronic hypoxia leads to reversible oligozoospermia (Verratti et al., 2008). Previously, it has been suggested that an HA sojourn may induce reversible spermatogenic and Leydig cell dysfunctions (Okumura et al., 2003). Recently, Verratti $V$ et al. found that a prolonged high altitude hiking leads to a reduction in human testicular volume (Verratti et al., 2017). So far, no study has investigated human testicular histological changes in males under HA hypoxic environment. Additionally, no study has compared the human semen parameters of males living in the hypoxic environments of plateaus and plains. To the best of our knowledge, this is the first large-scale comparative study on the semen quality of males who have been life-long residents of plateaus and plains.

\section{Native Tibetans had a lower NF\%, PR\%, VCL, VSL, total sperm number of PR, higher (PR+NP)\%, but similar total number of NF sperms}

First, we found that in the first 40 years of life, the incidence of teratozoospermia and asthenozoospermia was higher in males from HA areas than in those from plains. Further, we stratified the data by age and found that the incidence of teratozoospermia and asthenozoospermia in males residing in plateaus increased with age while the incidence of oligozoospermia did not change significantly. Normozoospermia remained stable with age in both the groups. Second, we studied the sperm parameters and stratified the data again according to age. Native Tibetans had a lower percentage of NF and PR, lower average value of VCL and VSL (a measure of cell vigor), lower total sperm number of PR, and higher total percentage of PR+NP than HCILP (Table 1). The higher the percentage of sperms with normal morphology, the faster the VSL and the higher the chance of natural pregnancy. Previous studies have reported that the percentage of normal morphological sperms is lower in males from HAs than in those from low altitudes, which is similar to our results (Gonzales, LozanoHernandez, Gasco, Gonzales-Castaneda, \& Tapia, 2012). Furthermore, we found that there was no significant difference in the total number of NF sperms between the two groups. Additionally, an exposure to high concentrations of lead ( $\mathrm{Pb}$ ) is associated with abnormal sperm morphology, poor acrosome alterations, and low fertilization rate (Godinez-Solis et al., 2019). Therefore, in addition to low temperature and high radiation, future studies should analyze whether there are any coexisting causes of abnormal sperm morphology, such as metal ion pollution and trace element deficiency, in plateau regions.

\section{Native Tibetans had higher LH, PRL, lower T, but similar FSH}

LH and FSH are gonadotropins secreted by the pituitary gland. The secretion of LH and FSH is stimulated by hypothalamic gonadotropin-releasing hormone (GnRH) (Sharma, Thaventhiran, Minhas, Dhillo, \& Jayasena, 2020). However, they have their own secretion mechanisms and are mutually regulated with other hormones secreted by the pituitary gland, which may be the cause of inconsistent responses of LH and FSH to hypoxia. T is the main androgen secreted by Leydig cells. The synthesis of $\mathrm{T}$ is regulated by $\mathrm{LH}$, and Leydig cells use cholesterol as a raw material to synthesize T using a series of enzymes in the mitochondria and endoplasmic reticulum. To date, few studies have reported the influence of HA hypoxic environment on male reproductive capacity, and most of these studies have compared the changes in the levels of some reproductive hormones in the blood of males who have entered Tibet for a short time. Hypoxia at HAs causes reversible adverse effects on the semen quality and reproductive hormones of males (Jankovic Velickovic \& Stefanovic, 2014). Liu X et al. showed that a simulated HA hypoxic environment can reduce the reproductive functions of adult male rats, expression of GnRH in the hypothalamus, and activity of the hypothalamic-pituitary-gonadal axis. Thus, serum T and free T levels decrease at HAs (Liu, Liu, \& Xu, 2020). Our study (Table 2) found that native Tibetans had higher LH levels and lower T levels than HCILP; however, no significant difference was observed in FSH levels between the two groups. The decrease in T level might lead to an increase in LH level through negative feedback. During aging, we found increased FSH level and stable LH, E2, and T levels in native Tibetans.

A previous study reported that PRL levels in males at 5,380 meters decrease first, but if they stay at this altitude for several months, the levels significantly increase (He et al., 2015). Another study confirmed that PRL levels are low at 4,844 $\mathrm{m}$ and increase at 6,022 m; however, the increase is not significant (von Wolff et al., 2018). Benso et al. found that the levels of PRL and progesterone increased after 7 weeks at 5,200 meters (Benso et al., 2007); however, Liu W et al. showed that after an intermittent hypobaric hypoxia exposure, serum progesterone levels in rats decrease (W. Liu et al., 2020). Our study showed that PRL levels were higher in males from Lhasa than in those from Shanghai, and the levels decreased with age. At the same time, progesterone levels were higher in HCILP than in native Tibetans, which was similar to the result of an animal model.

\section{Native Tibetans had lower E2, TSI, but higher E2/T}

Further, we found that E2 levels were lower in native Tibetans than in HCILP, and no significant change was observed in E2 levels in the two groups with aging. We then calculated TSI (total T to LH) and E2/T (E2 to total T) (Nassan et al., 2020). Males from plateaus had a lower TSI and higher E2/T. No significant change was observed in the E2/T and TSI of native Tibetans with aging, but HCILP showed a significant decrease in TSI with aging (Table 2). Such findings indicate that, on plateaus, each unit of LH that produced less T or more T was converted to E2. Many studies have shown that hypoxia can cause abnormalities in hypothalamic-pituitary functions, which are primarily located in the hypothalamic-pituitary-testicular axis (Fløe, Hilberg, Wijsenbeek, \& Bendstrup, 2017; Semple et al., 1984). Due to the obvious decrease in T levels, the substrate of E2, E2 decreases accordingly under hypoxia. A linear correlation exists between the decrease in T level and decrease of arterial oxygen partial pressure, which supports the hypothesis that hypoxia inhibits the 
hypothalamus-pituitary-testis axis. Some studies have shown that hypoxia can cause histological damage to the adrenal cortex (Gosney, $1984 a$; Stobdan et al., 2017) and testis (Gosney, 1984b; Steinfeld et al., 2018), and can weaken or slow down the secretory activities of gonadotropic cells (X. Liu et al., 2020), resulting in an insufficient production or decreased activity of E2 synthase and aromatase, which transforms androgen into E2, leading to low E2 levels. The decrease in E2 levels can reduce airway responsiveness and improve ventilation, and these changes are adaptive in HA populations to adapt to hypoxic environments and maintain homeostasis.

\section{Native Tibetans had higher DFI}

Examination of conventional semen parameters can reflect the semen quality in terms of sperm concentration and sperm vitality; however, the determination of sperm functions is limited. Thus, conventional semen parameters cannot directly reflect the sperm fertilization ability and impact on embryo development. DFI is one of the most common indicators for evaluating sperm fertility and predicting pregnancy outcome. During spermatogenesis, some factors, such as diseases, high fever, elevated testicular temperature, environmental toxicants, smoking, drugs, and aging, can cause sperm DNA damage through some mechanisms. Some studies have suggested that DFI is related to abnormal chromatin packaging, oxidative stress, telomere deficiency, and other factors (du Fossé, van der Hoorn, van Lith, le Cessie, \& Lashley, 2020). A study revealed that E2 partially protects against chronic intermittent hypoxia (CIH)-induced vascular injury through the regulation of the Trx-1/Txnip pathway (Lan et al., 2017). $\mathrm{ClH}$ exposure decreases $\mathrm{O}_{2}$ consumption and complex I activity and increases ROS production (Laouafa et al., 2019), and the increase in ROS production is the known cause of sperm DFI (Westerman, 2020). We found that the DFI of native Tibetans was significantly higher than that of HCILP, and the E2 levels were lower in native Tibetans than in HCILP. Sperm DFI was in the normal range in the plateau population but was significantly higher than that in HCILP (Table 3). Our results are consistent with those of animal models.

This study had several imitations. First, the incidence and grade of varicocele, testicular volume, status of seminal plasma germ infection (mycoplasma, chlamydia, human papilloma virus), metabolic parameters such as glycemia, insulin, insulin-resistance, body mass index, and the number of cigarette smokers (in case of smokers) were absent in the enrolled population. The study was retrospectively performed in our clinic, and the REH and semen parameters were based on a single measurement. Therefore, these data could have been potentially misclassified. However, previous studies have shown that a single semen sample may suffice for studies that aim to identify the mean differences in semen quality between males (Chiu et al., 2017; Stokes-Riner et al., 2007). Second, the abstinence time was self-reported; thus, it has some uncertainty. As a result, our study sample is not representative of the general healthy population. Third, life-long residency information is not feasible to exactly estimate the individual exposure to hypoxic environment. Fourth, due to the observational nature of the study design and reverse causation between hypoxic environment and sperm parameters, REH and DFI cannot be ruled out. These limitations may weaken the statistical power of our dataset and should be taken into account in future studies.

\section{Conclusion}

In summary, the present study showed that HA hypoxic environment may affect the hypothalamus-pituitary-testis axis of males, resulting in a decrease in the levels of T, E2, and TSI and an increase in the levels of LH and E2/T. No significant effect was observed on the total number of NF sperms. Although the total number of PR sperms decreased and DFI increased in the plateau population, they did not affect the sustained rapid growth of the plateau population. To the best of our knowledge, this is the first large-scale investigation on the changes in fertility in native Tibetans. Further, some interesting phenomena were also recognized. In the future, we need to design more rigorous control experiments to explain the molecular mechanism of sperm quality changes, REH changes, and increased DFI due to HA hypoxic environments.

\section{Abbreviations}

DFI: DNA fragmentation index

LH: luteinizing hormone

PR: progressive motility

NF: normal forms

WHO: World Health Organization

FSH: follicle-stimulating hormone

GnRH: gonadotropin-releasing hormone

PRL: prolactin

AO: acridine orange

E2: Estradiol

VCL: curvilinear velocity

VSL: straight-line (rectilinear) velocity

HCILP: Han Chinese individuals living in the plains 
REH: reproductive endocrine hormones

HA: high altitude

$\mathrm{T}$ : testosterone

TSI: testosterone secretion index

\section{Declarations}

\section{Availability of data and materials}

The datasets used during the current study are available from the corresponding author on reasonable request.

\section{Compliance with ethical standards}

\section{Ethical approval and consent to participate}

All procedures involving human participants were conducted in accordance with the ethical standards of the Ethics Committee of Shanghai General Hospital affiliated to Shanghai Jiao Tong University and Tibet Fukang Women and Children's Hospital (31660308) and the guidelines of the Declaration of Helsinki.

Consent for publication Not applicable

\section{Competing interests}

The authors declare that they have no competing interests.

\section{Funding}

Zhao Yi-Cheng Medical Science Foundation (ZYYFY2018031);

National Natural Science Foundation of China (31660308);

\section{Authors' contributions}

Xiaoqiang LIU, Xia LI, and Li LIU conceived the project. Yuxuan SONG, Jun LI, Yongtao NI, Min CHEN, and Jian-lin HU performed the experiment. Luobu Ouzhu, Zheng LI, and Feng LIU collected the samples. Benhong GU and Shangren WANG wrote the paper with help from all of the authors, and they are Co-first authors.

\section{Acknowledgements}

We thank the research assistants from the Laboratory of the Center of Urologic Medicine, Shanghai General Hospital Affiliated to Shanghai Jiao Tong University and Tibet Fukang Women and Children's Hospital for their help in carrying out some of the assays. We are grateful to Zijue Zhu for his help with the statistical analysis. The paper was edited by Wiley Editing Services.

\section{References}

Alharbi, M., Hamouche, F., Phillips, S., Kadoch, J. I., \& Zini, A. (2019). Use of testicular sperm in couples with SCSA-defined high sperm DNA fragmentation and failed intracytoplasmic sperm injection using ejaculated sperm. Asian J Androl. doi:10.4103/aja.aja_99_19 Benso, A., Broglio, F., Aimaretti, G., Lucatello, B., Lanfranco, F., Ghigo, E., \& Grottoli, S. (2007). Endocrine and metabolic responses to extreme altitude and physical exercise in climbers. Eur J Endocrinol, 157(6), 733-740. doi:10.1530/eje-07-0355 Childebayeva, A., Jones, T. R., Goodrich, J. M., Leon-Velarde, F., Rivera-Chira, M., Kiyamu, M., . . Bigham, A. W. (2019). LINE-1 and EPAS1 DNA methylation associations with high-altitude exposure. Epigenetics, 14(1), 1-15. doi:10.1080/15592294.2018.1561117 Chiu, Y. H., Edifor, R., Rosner, B. A., Nassan, F. L., Gaskins, A. J., Mínguez-Alarcón, L., . . Chavarro, J. E. (2017). What Does a Single Semen Sample Tell You? Implications for Male Factor Infertility Research. Am J Epidemiol, 186(8), 918-926. doi:10.1093/aje/kwx169 Cikutovic, M., Fuentes, N., \& Bustos-Obregón, E. (2009). Effect of intermittent hypoxia on the reproduction of rats exposed to high altitude in the Chilean Altiplano. High Alt Med Biol, 10(4), $357-363$. doi:10.1089/ham.2009.1035 Cooper, T. G., Noonan, E., von Eckardstein, S., Auger, J., Baker, H. W., Behre, H. M., . . Vogelsong, K. M. (2010). World Health Organization reference values for human semen characteristics. Hum Reprod Update, 16(3), 231-245. doi:10.1093/humupd/dmp048 Del Vento, F., Vermeulen, M., de Michele, F., Giudice, M. G., Poels, J., des Rieux, A., \& Wyns, C. (2018). Tissue Engineering to Improve Immature Testicular Tissue and Cell Transplantation Outcomes: One Step Closer to Fertility Restoration for Prepubertal Boys Exposed to Gonadotoxic Treatments. Int J Mol Sci, 19(1). doi:10.3390/ijms19010286 Du, B. X., Zheng, J. Z., Liu, B., Tao, F. Z., Fang, Y. K., Xu, W. L., \& Cui, Y. (2017). [Tongjingling improves sperm DNA integrity and reduces oxidative stress in the testis of experimental varicocele rats]. Zhonghua Nan Ke Xue, 23(11), 1025-1031. du Fossé, N. A., van der Hoorn, M. P., van Lith, J. M. M., le Cessie, S., \& Lashley, E. (2020). Advanced paternal age is associated with an increased risk of spontaneous miscarriage: a systematic review and meta-analysis. Hum Reprod Update, 26(5), 650-669. doi:10.1093/humupd/dmaa010 Eckes, L. (1976). [Altitude adaptation. IV. Fertility and reproduction at high altitudes]. Gegenbaurs Morphol Jahrb, 122(5), 761-770. Farias, J. G., Bustos-Obregón, E., Orellana, R., Bucarey, J. L., Quiroz, E., \& Reyes, J. G. (2005). Effects of chronic hypobaric hypoxia on testis histology and round spermatid oxidative metabolism. Andrologia, 37(1), 47-52. doi:10.1111/j.1439-0272.2004.00651.x Fløe, A., Hilberg, O., Wijsenbeek, M., \& Bendstrup, E. (2017). Erectile dysfunction is a common problem in interstitial lung diseases. Sarcoidosis Vasc Diffuse Lung Dis, 
34(4), 356-364. doi:10.36141/svdld.v34i4.5692 Godinez-Solis, Y., Solis-Heredia, M. J., Roa-Espitia, A., Parra-Forero, L. Y., Hernandez-Gonzalez, E. O., HernandezOchoa, I., \& Quintanilla-Vega, B. (2019). Low concentrations of lead decrease the sperm fertilization ability by altering the acrosome reaction in mice. Toxicol Appl Pharmacol, 380, 114694. doi:10.1016/j.taap.2019.114694 Gonzales, G. F., Lozano-Hernandez, R., Gasco, M., Gonzales-Castaneda, C., \& Tapia, V. (2012). Resistance of sperm motility to serum testosterone in men with excessive erythrocytosis at high altitude. Horm Metab Res, 44(13), 987-992. doi:10.1055/s0032-1321854 Gosney, J. R. (1984a). The effects of hypobaric hypoxia on the corticotroph population of the adenohypophysis of the male rat. J Pathol, 142(3), 163-168. doi:10.1002/path.1711420303 Gosney, J. R. (1984b). Effects of hypobaric hypoxia on the Leydig cell population of the testis of the rat. J Endocrinol, 103(1), 59-62. doi:10.1677/joe.0.1030059 Gu, B. H., Liu, F., Li, J., Zhu, Z. J., Ni, Y. T., Li, P., . . Li, Z. (2018). [Impact of plateau environment on seminal characteristics of native Tibetans and immigrated Tibetan Hans]. Zhonghua Nan Ke Xue, 24(1), 27-32. Guan, Y., Zheng, X., Yang, Z., \& Li, S. (2008). Change and significance of the expression of c-kit and SCF following recovery from unilateral testicular torsion in rats. Clin Invest Med, 31 (3), E98-e105. doi:10.25011/cim.v31i3.3466 He, J., Cui, J., Wang, R., Gao, L., Gao, X., Yang, L., . . Yu, W. (2015). Exposure to Hypoxia at High Altitude (5380 m) for 1 Year Induces Reversible Effects on Semen Quality and Serum Reproductive Hormone Levels in Young Male Adults. High Alt Med Biol, 16(3), $216-222$. doi:10.1089/ham.2014.1046 Jankovic Velickovic, L., \& Stefanovic, V. (2014). Hypoxia and spermatogenesis. Int Urol Nephrol, 46(5), 887-894. doi:10.1007/s11255-013-0601-1 Jorgensen, N., Joensen, U. N., Jensen, T. K., Jensen, M. B., Almstrup, K., Olesen, I. A., . . Skakkebaek, N. E. (2012). Human semen quality in the new millennium: a prospective cross-sectional population-based study of 4867 men. BMJ Open, 2(4). doi:10.1136/bmjopen-2012-000990 Lan, X. F., Zhang, X. J., Lin, Y. N., Wang, Q., Xu, H. J., Zhou, L. N., . . Li, Q. Y. (2017). Estradiol Regulates Txnip and Prevents Intermittent Hypoxia-Induced Vascular Injury. Sci Rep, 7(1), 10318. doi:10.1038/s41598-017-10442-7 Laouafa, S., Roussel, D., Marcouiller, F., Soliz, J., Gozal, D., Bairam, A., \& Joseph, V. (2019). Roles of oestradiol receptor alpha and beta against hypertension and brain mitochondrial dysfunction under intermittent hypoxia in female rats. Acta Physiol (Oxf), 226(2), e13255. doi:10.1111/apha.13255 Levine, H., Jørgensen, N., Martino-Andrade, A., Mendiola, J., Weksler-Derri, D., Mindlis, I., . . Swan, S. H. (2017). Temporal trends in sperm count: a systematic review and meta-regression analysis. Hum Reprod Update, 23(6), 646-659.

doi:10.1093/humupd/dmx022 Liao, W., Cai, M., Chen, J., Huang, J., Liu, F., Jiang, C., \& Gao, Y. (2010). Hypobaric hypoxia causes deleterious effects on spermatogenesis in rats. Reproduction, 139(6), 1031-1038. doi:10.1530/rep-09-0557 Liu, W., Pu, L., Deng, B., Xu, H., Wang, Z., Wang, T., ... Chen, Z. (2020). Intermittent hypobaric hypoxia causes deleterious effects on the reproductive system in female rats. Biomed Pharmacother, $130,110511$. doi:10.1016/j.biopha.2020.110511 Liu, X., Liu, S., \& Xu, C. (2020). Effects of leptin on HPG axis and reproductive function in male rat in simulated altitude of 5500 m hypoxia environment. Biochem Biophys Res Commun, 529(1), 104-111. doi:10.1016/j.bbrc.2020.05.194 Nassan, F. L., Jensen, T. K., Priskorn, L., Halldorsson, T. I., Chavarro, J. E., \& Jørgensen, N. (2020). Association of Dietary Patterns With Testicular Function in Young Danish Men. JAMA Netw Open, 3(2), e1921610. doi:10.1001/jamanetworkopen.2019.21610 Nikshad, A., Aghlmandi, A., Safaralizadeh, R., Aghebati-Maleki, L., Warkiani, M. E., Khiavi, F. M., \& Yousefi, M. (2021). Advances of microfluidic technology in reproductive biology. Life Sci, 265, 118767. doi:10.1016/j.Ifs.2020.118767 Okumura, A., Fuse, H., Kawauchi, Y., Mizuno, I., \& Akashi, T. (2003). Changes in male reproductive function after high altitude mountaineering. High Alt Med Biol, 4(3), 349-353. doi:10.1089/152702903769192304 Parraguez, V. H., Atlagich, M., Díaz, R., Cepeda, R., González, C., De los Reyes, M., . . Raggi, L. A. (2006). Ovine placenta at high altitudes: comparison of animals with different times of adaptation to hypoxic environment. Anim Reprod Sci, 95(1-2), 151-157.

doi:10.1016/j.anireprosci.2005.11.003 Qi, X., Cui, C., Peng, Y., Zhang, X., Yang, Z., Zhong, H., . . Su, B. (2013). Genetic evidence of paleolithic colonization and neolithic expansion of modern humans on the tibetan plateau. Mol Biol Evol, 30(8), 1761-1778. doi:10.1093/molbev/mst093 Salmasi, A. H., Beheshtian, A., Payabvash, S., Demehri, S., Ebrahimkhani, M. R., Karimzadegan, M., . . Dehpour, A. R. (2005). Effect of morphine on ischemia-reperfusion injury: experimental study in testicular torsion rat model. Urology, 66(6), 1338-1342. doi:10.1016/j.urology.2005.06.101 Semple, P. D., Beastall, G. H., Brown, T. M., Stirling, K. W., Mills, R. J., \& Watson, W. S. (1984). Sex hormone suppression and sexual impotence in hypoxic pulmonary fibrosis. Thorax, 39(1), 46-51.

doi:10.1136/thx.39.1.46 Sharma, A., Thaventhiran, T., Minhas, S., Dhillo, W. S., \& Jayasena, C. N. (2020). Kisspeptin and Testicular Function-ls it Necessary? Int J Mol Sci, 21(8). doi:10.3390/ijms21082958 Steinfeld, K., Beyer, D., Mühlfeld, C., Mietens, A., Eichner, G., Altinkilic, B., . . Middendorff, R. (2018). Low testosterone in ApoE/LDL receptor double-knockout mice is associated with rarefied testicular capillaries together with fewer and smaller Leydig cells. Sci Rep, 8(1), 5424. doi:10.1038/s41598-018-23631-9 Stobdan, T., Akbari, A., Azad, P., Zhou, D., Poulsen, O., Appenzeller, O., . . Haddad, G. G. (2017). New Insights into the Genetic Basis of Monge's Disease and Adaptation to High-Altitude. Mol Biol Evol, 34(12), 3154-3168. doi:10.1093/molbev/msx239 Stokes-Riner, A., Thurston, S. W., Brazil, C., Guzick, D., Liu, F., Overstreet, J. W., . . Swan, S. H. (2007). One semen sample or 2? Insights from a study of fertile men. J Androl, 28(5), 638-643. doi:10.2164/jandrol.107.002741 Thiersch, M., \& Swenson, E. R. (2018). High Altitude and Cancer Mortality. High Alt Med Biol, 19 (2), 116-123. doi:10.1089/ham.2017.0061 Verratti, V., Berardinelli, F., Di Giulio, C., Bosco, G., Cacchio, M., Pellicciotta, M., . . Tenaglia, R. (2008). Evidence that chronic hypoxia causes reversible impairment on male fertility. Asian J Androl, 10(4), 602-606. doi:10.1111/j.1745-7262.2008.00346.x Verratti, V., Tartaro, A., Falone, S., Pellegrini, M., Pelliccione, F., \& Di Giulio, C. (2017). Long Trekking Experience at High Altitude Causes Testicular Volumetric Reduction in Humans: Evidence Based on Magnetic Resonance Imaging. High Alt Med Biol, 18(2), 191-192. doi:10.1089/ham.2017.0034 von Wolff, M., Nakas, C. T., Tobler, M., Merz, T. M., Hilty, M. P., Veldhuis, J. D., . . Pichler Hefti, J. (2018). Adrenal, thyroid and gonadal axes are affected at high altitude. Endocr Connect, 7(10), $1081-1089$. doi:10.1530/EC-18-0242 Weitz, C. (1981). Fertility of Nepalese Sherpas at moderate altitudes: comparisons with high-altitude data. Ann Hum Biol, 8(4), 383385. doi:10.1080/03014468100005191 Westerman, R. (2020). Biomarkers for demographic research: sperm counts and other male infertility biomarkers. Biodemography Soc Biol, 65(1), 73-87. doi:10.1080/19485565.2019.1706150 Wu, T. (2001). The Qinghai-Tibetan plateau: how high do Tibetans live? High Alt Med Biol, 2(4), 489-499. doi:10.1089/152702901753397054 Zheng, S., Liu, Y., Li, P., \& Tian, H. (2019). Short-Term High-Altitude Exposure (3600 m) Alters the Type Distribution of Sperm Deformity. High Alt Med Biol, 20(2), 198-202. doi:10.1089/ham.2018.0133

\section{Tables}

Table 1 Comparison of semen parameters between native Tibetans and HCILP 


\begin{tabular}{|c|c|c|c|c|c|c|c|c|c|c|c|c|c|c|}
\hline \multirow[t]{2}{*}{ Parameter } & \multirow[t]{2}{*}{ nationality } & \multicolumn{3}{|c|}{ Total: $16<a g e<40$} & \multirow[t]{2}{*}{$\mathrm{P} 1$} & \multicolumn{3}{|c|}{ Young: age $\leq 30$} & \multirow[t]{2}{*}{ P2 } & \multicolumn{3}{|c|}{ Old: age >30 } & \multirow[t]{2}{*}{ P3 } & \multirow[t]{2}{*}{ p4 } \\
\hline & & p5 & p50 & p75 & & p5 & p50 & p75 & & p5 & p50 & p75 & & \\
\hline \multirow[t]{2}{*}{ Age(yr) } & 1 & 24.00 & 30.00 & 34.00 & 0.9913 & 23.00 & 28.00 & 29.00 & 0.6366 & 31.00 & 34.00 & 36.00 & 0.8763 & 0.00 \\
\hline & 2 & 24.00 & 30.00 & 34.00 & & 23.00 & 28.00 & 29.00 & & 31.00 & 34.00 & 36.00 & & 0.00 \\
\hline \multirow{2}{*}{$\begin{array}{l}\text { Semen } \\
\text { volume (ml) }\end{array}$} & 1 & 1.20 & 3.10 & 4.20 & 0.6705 & 1.20 & 3.10 & 4.30 & 0.4230 & 1.20 & 3.10 & 4.20 & 0.1328 & 0.83 \\
\hline & 2 & 1.10 & 3.10 & 4.00 & & 1.20 & 3.20 & 4.20 & & 1.00 & 3.00 & 4.00 & & 0.00 \\
\hline \multirow{2}{*}{$\begin{array}{l}\text { Sperm } \\
\text { concentration } \\
\text { (A) }\end{array}$} & 1 & 11.50 & 52.40 & 82.20 & 0.9233 & 10.90 & 53.90 & 82.55 & 0.5939 & 12.50 & 51.00 & 81.70 & 0.4955 & 0.53 \\
\hline & 2 & 10.45 & 51.79 & 85.15 & & 10.73 & 52.77 & 82.35 & & 9.71 & 51.16 & 88.10 & & 0.52 \\
\hline \multirow{2}{*}{$\begin{array}{l}\text { Total sperm } \\
\text { number (B) }\end{array}$} & 1 & 26.40 & 157.76 & 276.21 & 0.4450 & 26.41 & 158.66 & 275.86 & 0.6805 & 25.76 & 154.24 & 277.12 & 0.5123 & 0.57 \\
\hline & 2 & 22.47 & 151.65 & 277.16 & & 23.10 & 155.15 & 273.95 & & 20.16 & 146.28 & 277.89 & & 0.34 \\
\hline \multirow[t]{2}{*}{$\mathrm{NF}(\%)$} & 1 & 1.30 & 3.70 & 5.90 & 0.0000 & 1.10 & 3.90 & 6.20 & 0.0003 & 1.40 & 3.60 & 5.30 & 0.0000 & 0.06 \\
\hline & 2 & 0.00 & 6.00 & 11.00 & & 0.00 & 6.00 & 11.00 & & 0.00 & 6.00 & 11.00 & & 0.75 \\
\hline \multirow{2}{*}{$\begin{array}{l}\text { Total sperm } \\
\text { number of NF } \\
\text { (B) }\end{array}$} & 1 & 0.75 & 5.91 & 12.81 & 0.5232 & 0.78 & 6.07 & 12.93 & 0.5709 & 0.72 & 5.76 & 12.46 & 0.7358 & 0.22 \\
\hline & 2 & 0.00 & 6.58 & 21.13 & & 0.00 & 6.69 & 20.72 & & 0.00 & 6.41 & 21.39 & & 0.46 \\
\hline \multirow[t]{2}{*}{$P R+N P(\%)$} & 1 & 23.20 & 61.20 & 74.80 & 0.0000 & 23.20 & 61.65 & 74.55 & 0.0000 & 23.50 & 60.10 & 74.90 & 0.0000 & 0.28 \\
\hline & 2 & 12.00 & 51.56 & 66.45 & & 12.00 & 53.01 & 68.02 & & 11.48 & 50.13 & 64.49 & & 0.06 \\
\hline \multirow[t]{2}{*}{ PR\% } & 1 & 7.60 & 35.60 & 47.30 & 0.0000 & 6.90 & 36.15 & 47.50 & 0.0000 & 8.40 & 35.10 & 47.10 & 0.0000 & 0.36 \\
\hline & 2 & 9.13 & 41.12 & 54.45 & & 9.25 & 42.33 & 56.22 & & 8.47 & 39.84 & 52.56 & & 0.02 \\
\hline \multirow{2}{*}{$\begin{array}{l}\text { Total sperm } \\
\text { number of PR } \\
\text { (B) }\end{array}$} & 1 & 4.17 & 51.61 & 113.92 & 0.0084 & 3.52 & 52.45 & 113.10 & 0.0244 & 4.49 & 49.35 & 118.68 & 0.1340 & 0.40 \\
\hline & 2 & 3.78 & 59.63 & 125.56 & & 4.12 & 63.52 & 133.86 & & 3.36 & 54.47 & 117.58 & & 0.10 \\
\hline \multirow[t]{2}{*}{ VCL(mean, C) } & 1 & 23.20 & 36.10 & 41.30 & 0.0000 & 23.40 & 36.15 & 41.00 & 0.0000 & 23.20 & 36.10 & 41.60 & 0.0000 & 0.88 \\
\hline & 2 & 31.23 & 48.97 & 55.60 & & 32.15 & 49.48 & 56.29 & & 30.88 & 48.15 & 55.06 & & 0.02 \\
\hline \multirow[t]{2}{*}{ VSL(mean, C) } & 1 & 8.10 & 14.70 & 17.70 & 0.0000 & 7.90 & 14.70 & 17.80 & 0.0000 & 8.40 & 14.50 & 17.50 & 0.0000 & 0.71 \\
\hline & 2 & 19.14 & 31.52 & 37.07 & & 19.60 & 31.96 & 37.56 & & 18.60 & 30.82 & 36.55 & & 0.00 \\
\hline
\end{tabular}

1: native Tibetans, 1111 men for total, 584 and 527 for young and old, respectively ; 2: HCILP, 1687 men for total, 895 and 792 for young and old respectively. NF: normal forms; VCL: curvilinear velocity; VSL: straight-line velocity. A: million per ml; B: million per ejaculate; C: $\mu \mathrm{m} / \mathrm{s}$. P1: HCILP vs. native Tibetans of total; P2: HCILP vs. native Tibetans among the young group; P3: HCILP vs. native Tibetans among the old group. P4: comparison between the young and the old group of the same nationality.

\section{Table 2 Comparison of reproduction hormones between native Tibetans and HCILP}




\begin{tabular}{|c|c|c|c|c|c|c|c|c|c|c|c|c|}
\hline \multirow[t]{2}{*}{ Parameters } & \multirow[t]{2}{*}{ Nationality } & \multirow[t]{2}{*}{$\mathbf{N}$} & \multicolumn{2}{|c|}{$\begin{array}{l}\text { Total: } \\
16 \leq \text { age } \leq 40\end{array}$} & 1 & \multicolumn{2}{|c|}{$\begin{array}{l}\text { Young: } \\
16 \leq \text { age } \leq 30\end{array}$} & \multirow[t]{2}{*}{ P2 } & \multicolumn{2}{|c|}{ Old: 30 age $\leq 40$} & \multirow[t]{2}{*}{ P3 } & \multirow[t]{2}{*}{ P4 } \\
\hline & & & mean & sd & & mean & sd & & mean & & & \\
\hline \multirow[t]{2}{*}{ Age(yrs) } & 1 & 221 & 31.34 & 4.20 & 0.0001 & 27.77 & 2.08 & 0.0004 & 34.58 & 2.75 & 0.7540 & 0.0000 \\
\hline & 2 & 253 & 29.66 & 4.71 & & 26.57 & 2.71 & & 34.45 & 2.72 & & 0.0000 \\
\hline \multirow[t]{2}{*}{$\mathrm{E} 2$ (pmol/L) } & 1 & 221 & 103.82 & 45.92 & 0.0000 & 103.64 & 44.62 & 0.0000 & 103.97 & 47.25 & 0.0000 & 0.9874 \\
\hline & 2 & 253 & 146.01 & 39.73 & & 147.66 & 40.31 & & 143.44 & 38.87 & & 0.4672 \\
\hline \multirow{2}{*}{$\begin{array}{l}\text { Prog } \\
\text { (nmol/L) }\end{array}$} & 1 & 221 & 0.29 & 0.27 & 0.0000 & 0.29 & 0.15 & 0.0000 & 0.30 & 0.35 & 0.0000 & 0.1739 \\
\hline & 2 & 253 & 2.22 & 0.84 & & 2.33 & 0.92 & & 2.04 & 0.68 & & 0.0147 \\
\hline \multirow[t]{2}{*}{$\mathrm{FSH}(\mathrm{U} / \mathrm{L})$} & 1 & 221 & 4.13 & 2.55 & 0.0950 & 3.90 & 2.80 & 0.4281 & 4.34 & 2.30 & 0.3398 & 0.0091 \\
\hline & 2 & 253 & 3.82 & 2.35 & & 3.51 & 1.93 & & 4.31 & 2.83 & & 0.0355 \\
\hline \multirow[t]{2}{*}{$\mathrm{LH}(\mathrm{U} / \mathrm{L})$} & 1 & 221 & 4.94 & 2.12 & 0.0000 & 4.98 & 1.99 & 0.0000 & 4.91 & 2.25 & 0.0000 & 0.3872 \\
\hline & 2 & 253 & 3.29 & 1.43 & & 3.19 & 1.33 & & 3.44 & 1.55 & & 0.2396 \\
\hline \multirow{2}{*}{$\begin{array}{l}\text { Prol } \\
\text { (nmol/L) }\end{array}$} & 1 & 221 & 11.34 & 3.87 & 0.0000 & 11.91 & 3.75 & 0.0000 & 10.82 & 3.91 & 0.0000 & 0.0186 \\
\hline & 2 & 253 & 8.97 & 3.48 & & 9.29 & 3.66 & & 8.48 & 3.14 & & 0.1318 \\
\hline \multirow[t]{2}{*}{$\mathrm{T}(\mathrm{nmol} / \mathrm{L})$} & 1 & 221 & 4.90 & 1.96 & 0.0000 & 5.00 & 1.88 & 0.0000 & 4.80 & 2.03 & 0.0000 & 0.3257 \\
\hline & 2 & 253 & 14.36 & 5.24 & & 14.88 & 5.16 & & 13.54 & 5.29 & & 0.0227 \\
\hline \multirow[t]{2}{*}{$\mathrm{E} 2 / \mathrm{T}$} & 1 & 221 & 0.22 & 0.11 & 0.0000 & 0.21 & 0.10 & 0.0000 & 0.22 & 0.11 & 0.0000 & 0.6070 \\
\hline & 2 & 253 & 0.11 & 0.05 & & 0.11 & 0.05 & & 0.11 & 0.05 & & 0.0949 \\
\hline \multirow[t]{2}{*}{ TSI } & 1 & 221 & 33.45 & 22.86 & 0.0000 & 33.36 & 20.42 & 0.0000 & 33.53 & 24.95 & 0.0000 & 0.7022 \\
\hline & 2 & 253 & 145.48 & 73.41 & & 104.30 & 82.66 & & 134.30 & 71.92 & & 0.0321 \\
\hline
\end{tabular}

1: native Tibetans, 221 men for total, 105 and 116 for young and old, respectively ; 2: HCILP, 253 men for total, 154 and 99 for young and old respectively. P1: HCILP vs. native Tibetans of total; P2: HCILP vs. native Tibetans among the young group; P3: HCILP vs. native Tibetans among the old group. P4: comparison between the young and the old group of the same nationality. E2/T: the ratio of estradiol $(\mathrm{pg} / \mathrm{ml})$ to total testosterone(ng/dl), convert E2 to picograms per mililiter, divide by 3.671 , and convert T to nanograms per deciliter multiply by 0.0347 . TSI: the ratio of total testosterone(ng/dl) to $\mathrm{LH}$.

Table 3 Comparison of DFI between the native Tibetans and HCILP

\begin{tabular}{|c|c|c|c|c|c|c|c|c|c|c|c|c|c|c|c|c|}
\hline \multirow[t]{2}{*}{ Parameters } & \multirow[t]{2}{*}{ Nationality } & \multicolumn{4}{|c|}{ Total: $19<a g e<40$} & \multirow[t]{2}{*}{ P1 } & \multicolumn{3}{|c|}{ Young: age $\leq 30$} & \multirow{2}{*}{$\begin{array}{l}\text { P2 } \\
\text { p75 }\end{array}$} & \multicolumn{3}{|c|}{ Old: 30هage } & \multicolumn{3}{|c|}{ P3 } \\
\hline & & $\mathbf{N}$ & p5 & p50 & p75 & & $\mathbf{N}$ & p5 & p50 & & & $\mathbf{N}$ & p5 & p50 & p75 & \\
\hline \multirow[t]{2}{*}{ Age(yrs) } & 1 & 133 & 23.00 & 32.00 & 36.00 & 0.0204 & 43 & 21.00 & 25.00 & 28.00 & 0.0010 & 90 & 30.00 & 35.00 & 37.00 & 0. \\
\hline & 2 & 393 & 25.00 & 31.00 & 34.00 & & 159 & 23.00 & 27.00 & 28.00 & & 234 & 30.00 & 33.00 & 36.00 & \\
\hline \multirow[t]{2}{*}{ DFI(\%) } & 1 & 133 & 15.20 & 23.11 & 25.34 & 0.0000 & 43 & 13.06 & 21.89 & 24.69 & 0.0000 & 90 & 15.24 & 23.44 & 25.62 & 0. \\
\hline & 2 & 393 & 1.11 & 7.22 & 12.64 & & 159 & 1.18 & 7.21 & 12.64 & & 234 & 0.96 & 7.26 & 12.76 & \\
\hline
\end{tabular}

1: native Tibetans, 133 men for total, 43 and 90 for young and old, respectively ; 2: HCILP, 393 men for total, 159 and 234 for young and old respectively. P1: native Tibetans vs HCILP for total; P2: native Tibetans vs. HCILP among the young group; P3: native Tibetans vs HCILP among the old group; P4: comparison between the young and the old group of the same nationality. DFI: DNA fragmentation index.

\section{Figures}




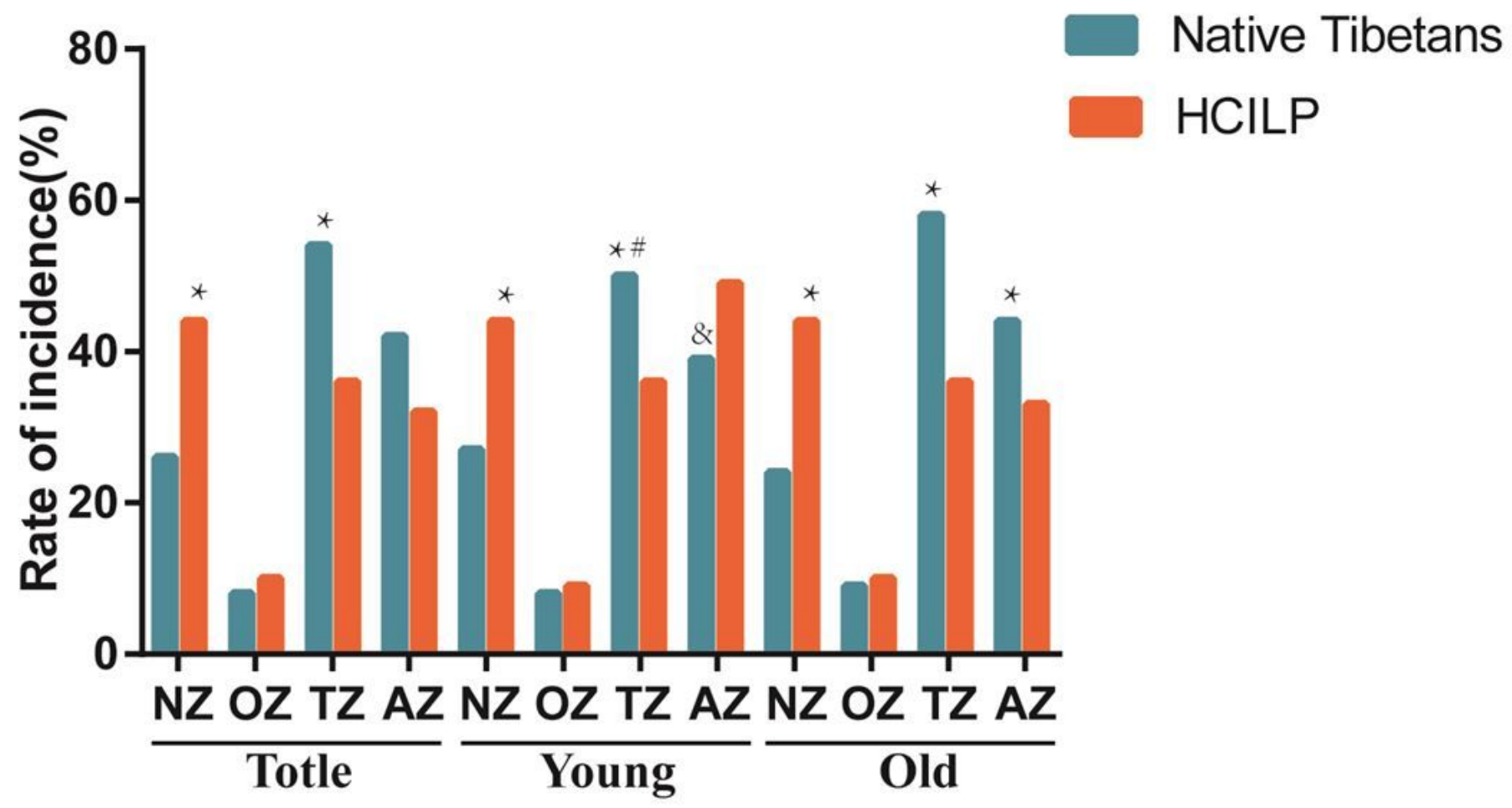

Figure 1

Prevalence of oligozoospermia, asthenozoospermia, or teratozoospermia in each group *: comparison between the native Tibetans and HCILP in total, $\mathrm{p} \|$ 0.001; \#: comparison between the young and the old males of native Tibetans, $\mathrm{p} \otimes 0.05$; \&: comparison between the young and the old males of HCILP, $\mathrm{p} \otimes$ 0.001. NZ: normozoospermia; OZ: Oligozoospermia; TZ: Teratozoospermia; AZ: Asthenozoospermia. 